Article

\title{
Structure of the Basal Edge Dislocation in $\mathrm{ZnO}$
}

\author{
Atsutomo Nakamura ${ }^{1, *(1)}$, Eita Tochigi ${ }^{2}$ (i), Ryota Nagahara ${ }^{1}$, Yuho Furushima ${ }^{1}$, Yu Oshima ${ }^{1}$ (i), \\ Yuichi Ikuhara ${ }^{2,3}$, Tatsuya Yokoi ${ }^{1}$ and Katsuyuki Matsunaga ${ }^{1,3}$ \\ 1 Department of Materials Physics, Nagoya University, Furo-cho, Chikusa-ku, Nagoya 464-8603, Japan; \\ nagahara.ryouta@e.mbox.nagoya-u.ac.jp (R.N.); furushima.yuuho@b.mbox.nagoya-u.ac.jp (Y.F.); \\ ooshima.yuu@f.mbox.nagoya-u.ac.jp (Y.O.); yokoi@mp.pse.nagoya-u.ac.jp (T.Y.); \\ kmatsunaga@nagoya-u.jp (K.M.) \\ 2 Institute of Engineering Innovation, University of Tokyo, 2-11-16 Yayoi, Bunkyo-ku, Tokyo 113-8686, Japan; \\ tochigi@sigma.t.u-tokyo.ac.jp (E.T.); ikuhara@sigma.t.u-tokyo.ac.jp (Y.I.) \\ 3 Nanostructures Research Laboratory, Japan Fine Ceramics Center, 2-4-1, Mutsuno, Atsuta-ku, \\ Nagoya 456-8587, Japan \\ * Correspondence: anaka@nagoya-u.jp; Tel.: +81-52-789-3366
}

Received: 19 January 2018; Accepted: 6 March 2018; Published: 8 March 2018

\begin{abstract}
Basal dislocations having a Burgers vector of $1 / 3<2 \overline{11} 0>$ in zinc oxide $(\mathrm{ZnO})$ with the wurtzite structure are known to strongly affect physical properties in bulk. However, the core structure of the basal dislocation remains unclear. In the present study, $\mathrm{ZnO}$ bicrystals with a $\{2 \overline{11} 0\} /<01 \overline{1} 0>2^{\circ}$ low-angle tilt grain boundary were fabricated by diffusion bonding. The resultant dislocation core structure was observed by using scanning transmission electron microscopy (STEM) at an atomic resolution. It was found that a basal edge dislocation in $\alpha$-type is dissociated into two partial dislocations on the (0001) plane with a separation distance of $1.5 \mathrm{~nm}$, indicating the glide dissociation. The Burgers vectors of the two partial dislocations were $1 / 3<1 \overline{1} 00>$ and $1 / 3<10 \overline{1} 0>$, and the stacking fault between the two partials on the (0001) plane has a formation energy of $0.14 \mathrm{~J} / \mathrm{m}^{2}$. Although the bicrystals have a boundary plane of $\{2 \overline{11} 0\}$, the boundary basal dislocations do not exhibit dissociation along the boundary plane, but along the (0001) plane perpendicular to the boundary plane. From DFT calculations, the stacking fault on the (0001) plane was found to be much more stable than that on $\{2 \overline{11} 0\}$. Such an extremely low energy of the (0001) stacking fault can realize transverse dissociation of the basal dislocation of $\mathrm{ZnO}$.
\end{abstract}

Keywords: zinc oxides ( $\mathrm{ZnO})$; wurtzite structure; line defects; low-angle grain boundaries; scanning transmission electron microscopy (STEM); generalized stacking fault (GSF) energy

\section{Introduction}

The wurtzite structure is a stable crystal structure for binary compound semiconducting materials such as $\mathrm{GaN}, \mathrm{AlN}, \mathrm{CdS}, \mathrm{ZnO}$, and so on. In these materials, $\mathrm{ZnO}$ is a representative wide and direct band gap semiconductor and has long been used as a main constituent material of varistors because of its highly nonlinear current-voltage characteristics [1-4]. In addition, $\mathrm{ZnO}$ has received broad interest due to its high electron mobility, high thermal conductivity and large exciton binding energy suitable for a variety of applications [1-3]. Although a large number of studies have been performed so far, most of them have aimed to control point defects and grain boundaries, both of which play a critical role in functional properties [1-6]. On the other hand, it is likely that atomic structures of dislocations, which dominate mechanical properties and also affect functional properties, have been poorly understood. This situation is also applied to the other wurtzite crystals.

Since the Burgers vector of the $1 / 3<2 \overline{11} 0>$ dislocation on the (0001) basal plane corresponds to the minimum translation of the wurtzite structure, the $<2 \overline{11} 0>$ slip on (0001) can work as an easy slip 
system in $\mathrm{ZnO}[7,8]$. It is interesting that such a basal dislocation in $\mathrm{ZnO}$ brings about localized energy levels within the band-gap, which may influence local optical properties [8]. It is also remarkable that hardness and flow deformation stresses of $\mathrm{ZnO}$ can be affected by light exposure [9]. However, atomic structures of basal dislocations in $\mathrm{ZnO}$ still remain unclear, as they have not been investigated using scanning transmission electron microscopy (STEM) with an atomic resolution.

There exist four candidates for core structures of the basal edge dislocation in $\mathrm{ZnO}$. This is because in the wurtzite structure the (0001) slip can occur on either of two inequivalent atomic planes [10-13], which are shown in Figure 1 as "glide type" and "shuffle type", and moreover, the [0001] and [0001] directions are not equivalent due to lack of inversion symmetry. In the case of the glide type, the basal edge dislocation will have its slip plane between the narrowly spaced $\mathrm{Zn}$ and $\mathrm{O}$ planes along [0001]. In this case, a basal dislocation has the potential to dissociate into two partial dislocations according to the Shockley partial reaction $[10,13]$. On the other hand, the shuffle type dislocation has its slip plane between the widely spaced $\mathrm{Zn}$ and $\mathrm{O}$ planes, where the basal dislocation cannot dissociate into partials because of the absence of stable stacking faults resulting from the crystal structure. Thus, two candidates of core structures are present due to their slip planes. Additionally, since there is no symmetry along [0001], two kinds of cores can be formed for both of the glide and shuffle type dislocations depending on whether an extra half plane of the dislocations is inserted toward [0001] or [0001] [13]. Although there are four candidates, the core structure of the basal edge dislocation has not, thus far, been experimentally characterized. Thus, it is of great importance to reveal a core structure of the basal edge dislocation for understanding an effect of dislocations on material properties in $\mathrm{ZnO}$.

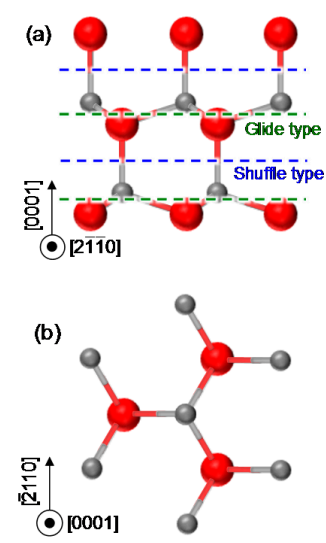

Figure 1. Schematic illustration showing the crystal structure of $\mathrm{ZnO}$ with the wurtzite structure. The arrangement of ions along [2 $\overline{11} 0]$ and [0001] are represented in $(\mathbf{a}, \mathbf{b})$, respectively. Large red circles correspond to oxygen ions while small gray circles do zinc ions. Locations of slip planes for glide type and shuffle type dislocations are indicated in (a) by dotted lines.

In the present study, therefore, we focus on the core structure of the basal edge dislocation in wurtzite $\mathrm{ZnO}$. Bicrystal experiments with artificial fabrication of a low-angle grain boundary are performed. The bicrystal experiment has proven to be an efficient method for evaluating atomic structures and properties of dislocations [14-17]. This is because a low-angle tilt grain boundary consists of periodically arranged edge dislocations, which have a Burgers vector perpendicular to the boundary plane. Accordingly, by fabricating a bicrystal with a controlled crystallographic relationship, periodical dislocations with a desirable Burgers vector can be produced at the boundary. A ZnO bicrystal with a $\{2 \overline{11} 0\} /<01 \overline{1} 0>$ low-angle tilt grain boundary was fabricated by diffusion bonding of two single crystals. In this case, basal edge dislocations that have a Burgers vector of $1 / 3<2 \overline{11} 0>$ should be periodically formed at the boundary. Resultant dislocation structures were characterized by transmission electron microscopy (TEM) and STEM. Moreover, formation energies of stacking faults in 
$\mathrm{ZnO}$ were evaluated by density functional theory (DFT) calculations to understand the origin of the observed atomic structure of the basal edge dislocation.

\section{Experimental Procedure}

Wurtzite $\mathrm{ZnO}$ single crystals grown by the hydrothermal synthesis were used to fabricate a bicrystal with a $\{2 \overline{11} 0\} /<01 \overline{1} 0>$ low-angle tilt grain boundary. Figure 2a shows schematic illustrations of two pieces of the single-crystal plates used for fabricating the bicrystal. Each single crystal plate

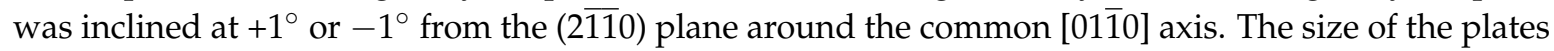
was set as $10 \times 5 \times 1 \mathrm{~mm}^{3}$, and their surfaces were polished by a diamond slurry and a colloidal silica to achieve a mirror finish. The two single-crystal plates were then joined by diffusion bonding at $1120^{\circ} \mathrm{C}$ in air for $10 \mathrm{~h}$ under a uniaxial load of $25 \mathrm{~N}$. Owing to the $1^{\circ}$ inclination of the crystallographic orientation of the individual grains, the tilt misorientation angle of $2^{\circ}$ was introduced at the bonding interface. As a result, a $\mathrm{ZnO}$ bicrystal with a $\{2 \overline{11} 0\} /<01 \overline{1} 0>2^{\circ}$ tilt grain boundary was fabricated as shown in Figure $2 \mathrm{~b}$. Here, the relation between the spacing between periodic basal dislocations $d$ and the misorientation angle $\theta$ in a low-angle tilt grain boundary is given by the equation of $\theta=b / d$, where $b$ is the magnitude of the edge component of the Burgers vector of boundary dislocations, according to the Frank's formula [18]. Figure 2c shows the $d, b$ and $\theta$ at the boundary. Therefore, if $\theta=2^{\circ}$ and $b=0.325 \mathrm{~nm}(|1 / 3<2 \overline{11} 0>|)[1]$ are substituted in the above equation, the spacing $d$ is obtained as $9.3 \mathrm{~nm}$.

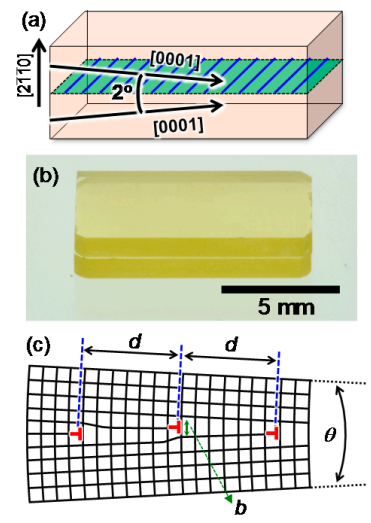

Figure 2. Schematics and optical micrograph of the fabricated $\mathrm{ZnO}$ bicrystal with a $(2 \overline{11} 0) /[01 \overline{1} 0] 2^{\circ}$ tilt grain boundary. (a) Schematic illustration showing the crystallographic orientations of two pieces of used $\mathrm{ZnO}$ single-crystal plates. (b) A fabricated $\mathrm{ZnO}$ bicrystal. (c) Schematic illustration showing periodic dislocations at the boundary.

Since $\mathrm{ZnO}$ has a polar crystal structure along the [0001] direction according to the wurtzite structure, the crystal lattice structure along [0001] differs from the one along the opposite direction of [0001] . It should be mentioned that the [0001] directions in the fabricated bicrystals were set up to face into the bonding interface as shown in Figure 2a. In this case, an extra half plane of dislocations introduced at the boundary is inserted toward [0001] when viewed from the dislocation cores. This type of dislocation is called " $\alpha$-dislocation", while the other type of dislocation with the extra half plane toward [0001] is called " $\beta$-dislocation" [13]. There is a difference between $\alpha$-dislocation and $\beta$-dislocation in terms of atomic species at the edge of extra half plane; $\mathrm{Zn}$ or O.

The grain boundary of bicrystals thus fabricated was observed by TEM and STEM. Specimens for the observations were prepared using a standard technique involving mechanical grinding to a thickness of $60 \mu \mathrm{m}$, dimpling to a thickness of about $30 \mu \mathrm{m}$ and ion beam milling to electron transparency. Observations were conducted by a conventional TEM (Hitachi H-800, 200 kV, Japan) and an atomic resolution STEM (JEOL JEM-ARM200F, 200 kV, Japan). 


\section{DFT Calculations}

Stacking fault energies in $\mathrm{ZnO}$ were calculated using DFT calculations based on the projector augmented wave (PAW) method as implemented in VASP code $[19,20]$. In the PAW potentials, Zn $3 d 4 s$ and $\mathrm{O} 2 s 2 p$ electrons were treated as valence electrons. The generalized gradient approximation (GGA) parameterized by Perdew, Burke and Ernzerhof was used for the exchange-correlation term [21]. To correct for the on-site Coulomb interaction of the $3 d$ orbitals of $\mathrm{Zn}$ atoms, the rotationally invariant $+U$ method [22] was applied with $U=8 \mathrm{eV}$ [23]. Wavefunctions were expanded by plane waves with a cut-off energy of $600 \mathrm{eV}$.

Since the glide type dislocation dissociates into two partial dislocations according to the Shockley reaction of $1 / 3<2 \overline{11} 0>\rightarrow 1 / 3<10 \overline{1} 0>+1 / 3<1 \overline{1} 00>$, the sheared structure along $<10 \overline{1} 0>$ should be considered for the stacking faults on (0001). Here, the (0001) atomic layers consist of like ions, and accordingly, each (0001) plane becomes a polar plane. Moreover, the $<10 \overline{1} 0>$ direction does not have mirror symmetry. In order to calculate stacking fault energies on (0001), therefore, 72-atom supercells containing three slabs with twelve $\{0001\}$ atomic layers each were employed. In this case, no vacuum layer was involved in the supercells, because the polar surfaces of the atomic slabs may induce spurious electric-dipole interactions normal to the slab surfaces. It is noted that, when three slabs are relatively displaced by a same amount toward three different $<10 \overline{1} 0>$ directions, three stacking faults then produced in the supercells are equivalent to one another [24,25]. Brillion zone integration was performed with an $8 \times 8 \times 1 k$-point mesh for the stacking faults on (0001). In contrast, supercells containing two atomic slabs with a vacuum layer of $1.6 \mathrm{~nm}$ were employed to calculate stacking fault

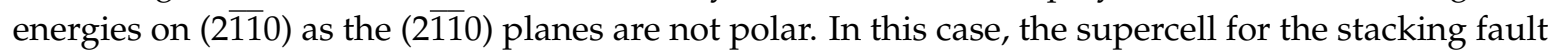
on (2110) contains 40 atoms (corresponding to 10 atomic layers) and a stacking fault. Brillion zone integration was performed with a $6 \times 6 \times 1 \mathrm{k}$-point mesh for the stacking faults on (2110). Structure optimizations for all the calculations were conducted until residual forces of atoms reached to less than $0.01 \mathrm{eV} / \AA$.

\section{Results and Discussion}

Figure 3 shows a typical bright field TEM image of the $(2 \overline{11} 0) /[01 \overline{1} 0] 2^{\circ}$ tilt grain boundary taken along [0110]. As can be seen from the image, the dot-like contrasts were distinctly observed at the boundary, which should be caused by boundary dislocations. A spacing between the contrasts was estimated to be about $9 \mathrm{~nm}$, which is in good agreement with the expected spacing between dislocations of $\boldsymbol{b}=1 / 3[2 \overline{11} 0]$ at the boundary $(9.3 \mathrm{~nm}$, see Section 2). Diffraction spots originating from both of the two grains around the boundary were separated from each other by approximately $2^{\circ}$, which coincides with the designed tilt angle. Thus, the $(2 \overline{11} 0) /[01 \overline{1} 0] 2^{\circ}$ tilt grain boundary was successfully fabricated, and it was suggested that $1 / 3[2 \overline{11} 0]$ basal dislocations were periodically introduced at the boundary.

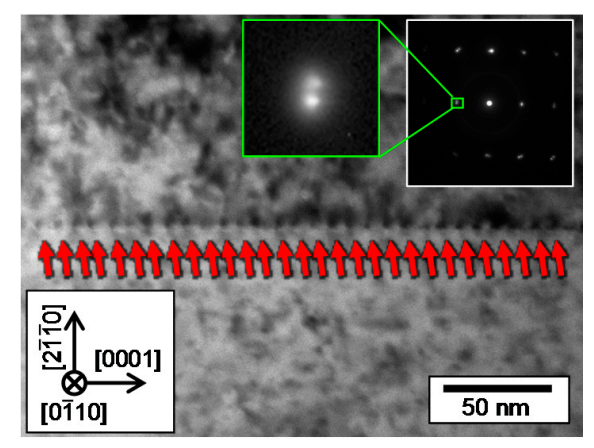

Figure 3. A typical bright field TEM image of the $(2 \overline{11} 0) /[01 \overline{1} 0] 2^{\circ}$ tilt grain boundary taken along [0110]. A corresponding selected-area diffraction pattern is inset right above. 
Figure 4a shows a typical high-angle annular dark field (HAADF) STEM image taken from one of the dislocations at the grain boundary. In this image, the bright spots correspond to Zn columns. It can be seen that two lattice discontinuities clearly appear in the Burgers circuits on the image. This means that a dislocation dissociates into two partial dislocations. Here, the spacings of the bright points on the HAADF-STEM image correspond to the magnitude of 1/2[0001] along [0001] and the magnitude of 1/6[2110] along [2110]. Accordingly, the Burgers circuits on the image show that the each partial dislocation has the same edge component of $1 / 6[2110]$, and thus the total edge component is $1 / 3[2 \overline{11} 0]$. It was confirmed that the periodic dislocations in Figure 3 are characterized as basal dislocations represented by the same Burgers circuits in Figure 4a. Figure $4 \mathrm{~b}$ shows an inverse Fast-Fourier-Transformed (FFT) image reconstructed from a mask-applied FFT image of the area shown in Figure 4a. The separation distance between the two partial dislocations is $1.5 \mathrm{~nm}$ along [2110], while the two seem to be adjacently located along [0001]. It can thus be said that the basal edge dislocation

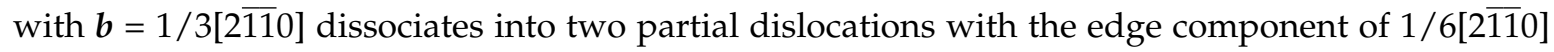
along (0001). Such a feature of the basal dislocation dissociating into two partials ensures that the basal dislocations at the fabricated boundary have the core structure of the glide-type dislocation. In this case, each partial dislocation should have the Burgers vector of $\boldsymbol{b}_{1}=1 / 3[10 \overline{1} 0]$ or $\boldsymbol{b}_{\mathbf{2}}=1 / 3[1 \overline{1} 00]$, according to the Shockley partial reaction of the glide type basal dislocation. In fact, the edge component of $1 / 6[2 \overline{11} 0]$ corresponds to the projection vector of the partial dislocations with $\boldsymbol{b}_{\mathbf{1}}$ and $\boldsymbol{b}_{\mathbf{2}}$ onto the (0110) plane. This also supports that the basal dislocations dissociate into two partials according to the Shockley partial reaction.

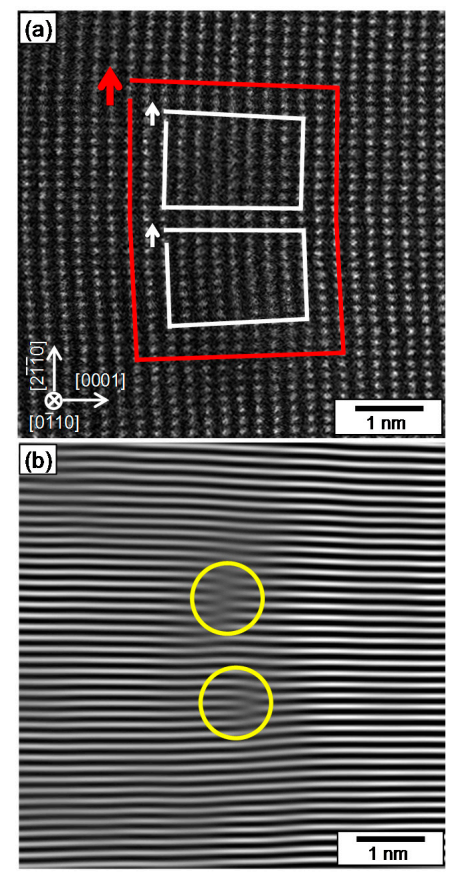

Figure 4. (a) A typical HAADF-STEM image taken from one of the contrasts on the image of Figure 3. Burgers circuit for a basal edge dislocation is drawn by red color line while Burgers circuits for two partial dislocations are drawn by white color lines. (b) The inverse Fast-Fourier-Transformed (FFT) image reconstructed from a mask-applied FFT image of (a). Yellow circles indicate the positions of cores of partial dislocations.

When a dislocation dissociates into two partial dislocations, a stacking fault is formed between the partials. In the present case, the stacking fault is formed along the (0001) plane perpendicularly to the (2110) boundary plane. The fault vector of the stacking fault corresponds to the Burgers vector of the partial dislocation of $1 / 3[10 \overline{1} 0]$ ( or $1 / 3[1 \overline{1} 00]$ ). Here, the separation distance between partials is 
determined by a balance of the two forces acting in the dissociated dislocation, that is, the repulsive elastic force between partial dislocations and the attractive force due to the stacking fault energy. According to the Peach-Koehler equation [10], the balance in the present dissociated basal dislocation can be expressed by the following equation,

$$
\gamma=\frac{\mu b_{\mathrm{p}}{ }^{2}(2+v)}{8 \pi r(1-v)}
$$

where $\gamma$ is the stacking fault energy, $\mu$ is the shear modulus (44.3 GPa [26]), $v$ is the Poisson' ratio $(0.3177$ [26] $), b_{\mathrm{p}}$ is the magnitude of Burgers vectors of the partial dislocations and $r$ is the separation distance. See Supplementary Materials for the derivation of Equation (1). By substituting $1.5 \mathrm{~nm}$ for $r$ in Equation (1), the formation energy of the (0001) stacking fault with the fault vector of 1/3[1010] was estimated to be about $0.14 \mathrm{~J} / \mathrm{m}^{2}$. It should be mentioned that the equation is based on a conventional elastic theory for an isotropic elastic medium. Accordingly, the estimation may slightly lose accuracy due to the anisotropic elasticity of $\mathrm{ZnO}$. Here, the ratio of $c_{44}$ to $\left(c_{11}-c_{12}\right) / 2$ in $\mathrm{ZnO}$ was calculated to be 0.96 according to the elastic constants in the former report [26]. Since the value is close to $1, \mathrm{ZnO}$ seemingly exhibits almost isotropic elasticity.

Figure $5 \mathrm{a}, \mathrm{b}$ shows schematic illustrations of a dissociated dislocation and dissociated dislocations array at the boundary, respectively. The stacking sequence of the (2110) plane along [2110] in $\mathrm{ZnO}$ corresponds to $A B A B \ldots$, as shown in (a). Figure $5 \mathrm{~b}$ explains the periodic formation of dissociated basal dislocations and stacking faults between the two partial dislocations. In general, dislocations at symmetrical tilt grain boundaries tend to dissociate on the boundary plane [16,17] as shown in Figure $5 c$. This is believed to be due to the fact that total elastic energy derived from dislocations array is minimized when the dislocations are located in such a linear arrangement according to the elastic theory [10]. Accordingly, it is noteworthy that the dislocations dissociated perpendicular to the boundary plane as shown in Figure $5 b$. Here, a lower stacking fault energy makes a longer separation distance at a dissociated dislocation according to the Peach-Koehler equation [10]. In addition, two partial dislocations with a longer separation distance do not suffer excess elastic energies so much as those with a shorter separation distance since their excess elastic energies are accommodated with increasing separation distance. It is thus suggested that stacking fault energy in $\mathrm{ZnO}$ should be relatively lower on the (0001) basal plane than the (2110) boundary plane, resulting in the dissociation on (0001).

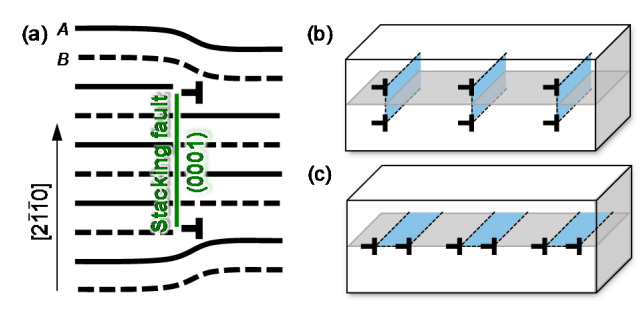

Figure 5. (a) Schematic illustration showing the observed structure of a dissociated basal dislocation. (b) Schematic illustration showing an array of the dissociated basal dislocations at the boundary. (c) Schematic illustration of usual structure of boundary dislocations, where dislocations dissociate on the boundary plane.

In order to confirm such a difference in stacking fault energy, DFT calculations were made for stacking faults on different planes of (0001) and (2110). Figure 6a,b shows a schematic of the [10 $\overline{1} 0]$ vector on the (0001) plane and the energy curve of the stacking faults along [1010] on the (0001) plane in $\mathrm{ZnO}$. The $u /\left|\boldsymbol{b}_{\mathrm{m}}\right|$ is employed as the horizontal axis in the energy curve, where $\boldsymbol{b}_{\mathrm{m}}$ is [1010] and $u$ is the displacement. The energies gradually increase up to the local maximum at $u /\left|\boldsymbol{b}_{\mathrm{m}}\right|=1 / 6$ and inversely decrease to the local minimum at $u /\left|\boldsymbol{b}_{\mathbf{m}}\right|=1 / 3$. Then, they again rapidly increase up to $u /\left|\boldsymbol{b}_{\mathrm{m}}\right|=2 / 3$ and inversely decrease to the initial state at $u /\left|\boldsymbol{b}_{\mathrm{m}}\right|=1$. The value at the local 
minimum of $u /\left|\boldsymbol{b}_{\mathbf{m}}\right|=1 / 3$ represents the energy of the stacking fault formed with the $1 / 3[10 \overline{1} 0]$ partial dislocation, which is the Shockley partial of the glide type basal dislocation. It can be said that it is energetically favorable for a glide-type basal dislocation to dissociate into two Shockley partials along (0001).
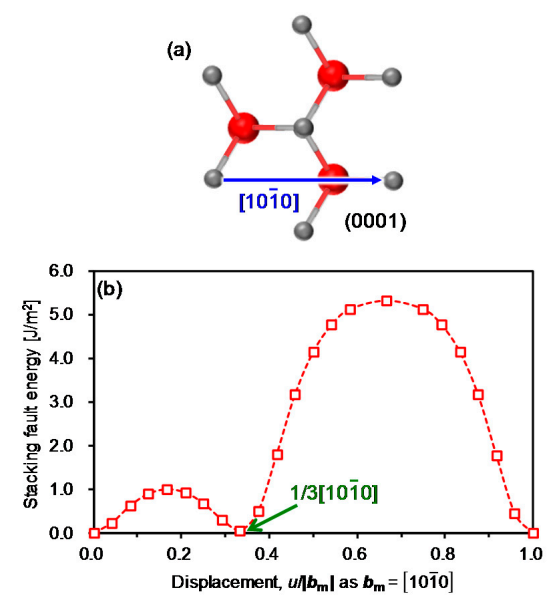

Figure 6. (a) Schematic illustration showing the [1010] vector on (0001). (b) Stacking fault energies along [1010] on (0001) in $\mathrm{ZnO}$. It can be seen that the energy indicates the local minimum at the displacement of $1 / 3[10 \overline{1} 0]$.

Figure $7 \mathrm{a}, \mathrm{b}$ shows the perfect crystal structure and the relaxed atomic structure of the stacking fault at $u /\left|\boldsymbol{b}_{\mathrm{m}}\right|=1 / 3$. It was found that the relaxed stacking fault structure in Figure $7 \mathrm{~b}$ is very close to the rigidly sheared atomic structure and individual atoms did not shift distinctly from the original positions before structure optimization. This also ensures the rather low energy of the stacking fault. The calculated stacking fault energy of $73 \mathrm{~mJ} / \mathrm{m}^{2}$ is in good agreement with that of $56 \mathrm{~mJ} / \mathrm{m}^{2}$ in the previous study [27]. It should be mentioned that the calculated value of the stacking fault energy at $u /\left|\boldsymbol{b}_{\mathrm{m}}\right|=1 / 3$ are $67 \mathrm{~mJ} / \mathrm{m}^{2}$ lower than the experimental value of $0.14 \mathrm{~J} / \mathrm{m}^{2}$, which was estimated from the separation distance between partial dislocations. Although the difference between the theoretical and experimental stacking fault energies is not distinct in terms of the absolute value, such a difference was also reported in $\mathrm{Al}_{2} \mathrm{O}_{3}[28,29]$. This difference may be brought about by temperature-dependent effects such as vibrational entropy.

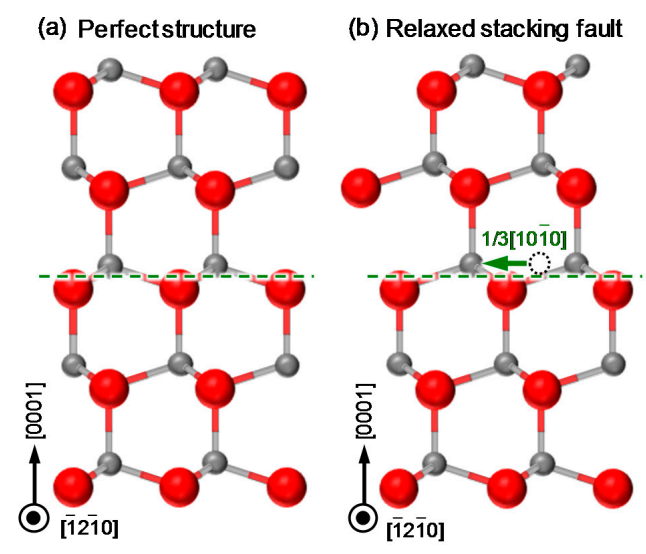

Figure 7. Schematic illustrations showing atomic structure of a stacking fault on (0001). (a) Perfect structure of $\mathrm{ZnO}$ along [1]210]. (b) A relaxed stacking fault structure with the fault vector of $1 / 3[10 \overline{1} 0]$ after structure optimization. 
Figure $8 \mathrm{a}, \mathrm{b}$ shows a schematic of the [0001] and [0110] vectors on $(2 \overline{110})$ and stacking fault energies on (2110) as functions of $u /\left|\boldsymbol{b}_{\mathrm{m}^{\prime}}\right|$ and $u /\left|\boldsymbol{b}_{\mathrm{c}}\right|$, where $\boldsymbol{b}_{\mathrm{m}^{\prime}}=[01 \overline{1} 0]$ and $\boldsymbol{b}_{\mathrm{c}}=[0001]$, respectively. It should be noticed that the stacking faults on (2110) take the minimum energy of $0.40 \mathrm{~J} / \mathrm{m}^{2}$ at the center of the energy map in (b), where the fault vector is $1 / 2[01 \overline{1} 1]$. Here, the $1 / 2[01 \overline{1} 1]$ dislocations cannot be induced by dissociation of a basal dislocation because the size of $1 / 2[01 \overline{1} 1]$ is larger than that of $1 / 3<2 \overline{11} 0>$. In addition, the stacking fault on (2110) with the fault vector of $1 / 3[10 \overline{1} 0]$ due to the Shockley partial reaction has an energy of over $2.4 \mathrm{~J} / \mathrm{m}^{2}$, as shown in Figure $8 \mathrm{c}$. Such a stacking fault energy is close to twice the ( $2 \overline{11} 0$ ) surface energy (about $2.7 \mathrm{~J} / \mathrm{m}^{2}$ ). Moreover, the value of $2.4 \mathrm{~J} / \mathrm{m}^{2}$ is about 33 times higher than the stacking fault energy on (0001), which was estimated to be $73 \mathrm{~mJ} / \mathrm{m}^{2}$ in the present calculation. These indicate that such a stacking fault cannot be stably formed on the (2110) plane, and thus the basal dislocation does not show the Shockley partial reaction along the (2110) boundary plane. Instead, the basal dislocation can be dissociated into two partials with the rather stable stacking fault on the (0001) plane, as observed experimentally.

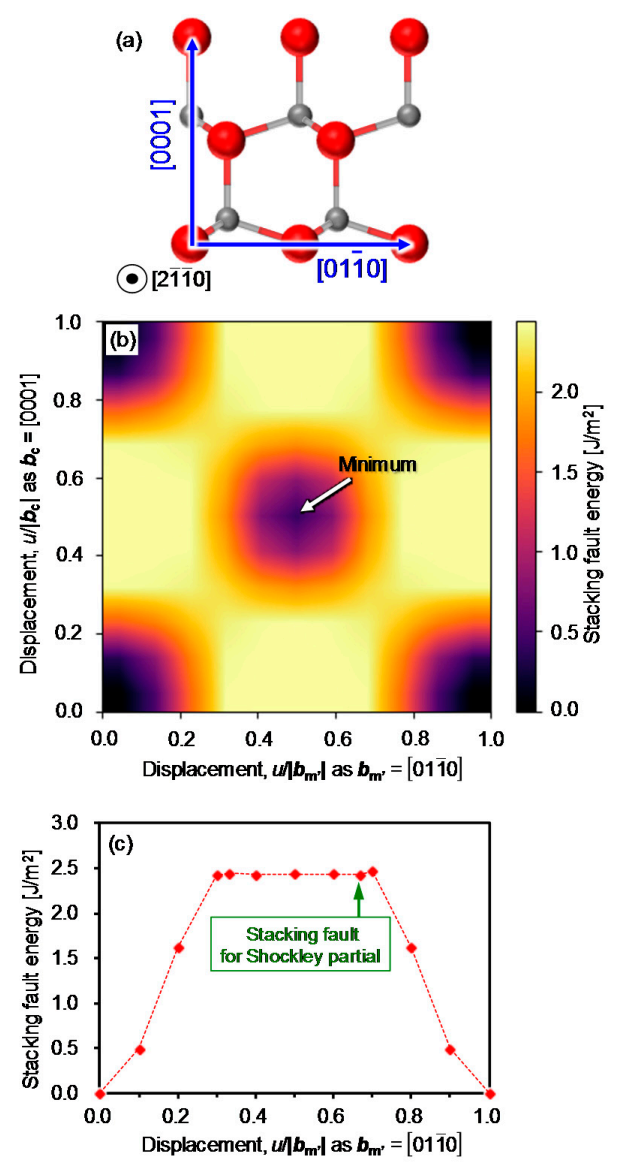

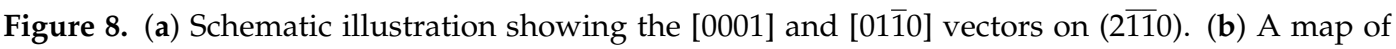
stacking fault energies on $(2 \overline{11} 0)$ in $\mathrm{ZnO}$. This map indicates the minimum value of $0.40 \mathrm{~J} / \mathrm{m}^{2}$ at the

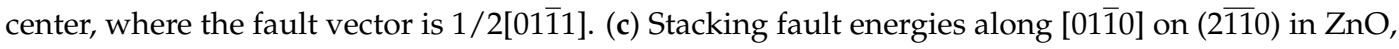
where the fault vector does not have the component along [0001]. Here, the (2110) stacking fault with the $2 / 3[01 \overline{1} 0]$ fault vector indicated by the green arrow in (c) is the same as that with the $1 / 3[0 \overline{1} 10]$ fault vector. In addition, owing to the symmetry of the wurtzite crystal structure, the (2110) stacking fault with the $1 / 3[0 \overline{1} 10]$ fault vector is equivalent to the $(2 \overline{11} 0)$ stacking fault with the $1 / 3[10 \overline{0} 0]$ fault vector, which corresponds to a Burgers vector of the Shockley partials in ZnO. Eventually, the energy of the stacking fault with the 2/3[0110] fault vector in (c) represents the energy of a stacking fault structure

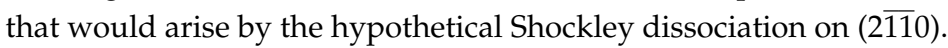




\section{Conclusions}

The $(2 \overline{110}) /[01 \overline{1} 0] 2^{\circ}$ tilt grain boundary in wurtzite $\mathrm{ZnO}$ was observed by using atomic-resolution STEM in order to investigate the core structure of the $1 / 3<2 \overline{11} 0>$ basal edge dislocation in $\alpha$-type. It was found that the basal edge dislocation in $\mathrm{ZnO}$ dissociates into two partial dislocations along (0001) with a separation distance of $1.5 \mathrm{~nm}$. This indicates that the basal edge dislocation in $\mathrm{ZnO}$ has a core structure of the glide-type dislocation, where the Burgers vector of the two partial dislocations should be $1 / 3<10 \overline{1} 0>$ and $1 / 3<1 \overline{1} 00>$. Applying the separation distance of $1.5 \mathrm{~nm}$ to the equation based on an elastic theory, the stacking fault energy on (0001) was experimentally estimated to be $0.14 \mathrm{~J} / \mathrm{m}^{2}$. Although the bicrystals have the $\{2 \overline{11} 0\}$ boundary plane, the basal dislocations do not exhibit dissociation along the boundary plane but dissociate along the (0001) basal plane perpendicular to the boundary plane. From DFT calculations, the stacking fault on the (0001) plane was found to be much more stable than that on $\{2 \overline{11} 0\}$. It is suggested that such an extremely low energy of the (0001) stacking fault causes dissociation of the basal dislocation on the (0001) plane.

Supplementary Materials: Supplementary texts and figures on the derivation of the Equation (1) and the initial and relaxed structures of supercells for the DFT calculations are available online at www.mdpi.com/2073-4352/8/ 3/127/s1.

Acknowledgments: The authors acknowledge I. Matsukura for technical assistance with TEM samples preparation. This work was mainly supported by a Grant-in-Aid for Scientific Research on Innovative Areas "Nano Informatics" (grant numbers JP25106002 and JP25106003) from Japan Society for the Promotion of Science (JSPS). A part of this study was supported by JSPS KAKENHI Grant Numbers JP15H04145, JP15K20959, JP17H06094, JP17K18982 and JP17K18983. Additionally, this work was partly supported by Nanotechnology Platform Program (Advanced Characterization Nanotechnology Platforms of Nagoya Univ. and Univ. of Tokyo) of the Ministry of Education, Culture, Sports, Science and Technology (MEXT), Japan.

Author Contributions: Atsutomo Nakamura and Katsuyuki Matsunaga conceived the research idea, and Atsutomo Nakamura designed the experiments; Atsutomo Nakamura and Eita Tochigi performed the experiments; Yuho Furushima, Yu Oshima and Atsutomo Nakamura analyzed the data; Ryota Nagahara and Atsutomo Nakamura performed the theoretical calculations; Katsuyuki Matsunaga, Tatsuya Yokoi and Yuichi Ikuhara advised the experiments and calculations; All the authors discussed the results and wrote the paper.

Conflicts of Interest: The authors declare no conflict of interest.

\section{References}

1. Pearton, S.J.; Norton, D.P.; Ip, K.; Heo, Y.W.; Steiner, T. Recent progress in processing and properties of ZnO. Prog. Mater. Sci. 2005, 50, 293-340. [CrossRef]

2. Janotti, A.; Van de Walle, C.G. Fundamentals of zinc oxide as a semiconductor. Rep. Prog. Phys. 2009, 72, 126501. [CrossRef]

3. Klingshirn, C.F.; Meyer, B.K.; Waag, A.; Hoffmann, A.; Geurts, J. Zinc Oxide: From Fundamental Properties Towards Novel Applications, 2010th ed.; Springer: Berlin/Heidelberg, Germany, 2010.

4. Sato, Y.; Yamamoto, T.; Ikuhara, Y. Atomic Structures and Electrical Properties of ZnO Grain Boundaries. J. Am. Ceram. Soc. 2007, 90, 337-357. [CrossRef]

5. Oba, F.; Nishitani, S.R.; Isotani, S.; Adachi, H.; Tanaka, I. Energetics of native defects in ZnO. J. Appl. Phys. 2001, 90, 824-828. [CrossRef]

6. Oba, F.; Ohta, H.; Sato, Y.; Hosono, H.; Yamamoto, T.; Ikuhara, Y. Atomic structure of [0001]-tilt grain boundaries in ZnO: A high-resolution TEM study of fiber-textured thin films. Phys. Rev. B 2004, 70, 125415. [CrossRef]

7. Osipiyan, Y.A.; Smirnova, I.S. Perfect dislocations in the wurtzite lattice. Phys. Status Solidi 1968, 30, 19-29. [CrossRef]

8. Ohno, Y.; Koizumi, H.; Taishi, T.; Yonenaga, I.; Fujii, K.; Goto, H.; Yao, T. Optical properties of dislocations in wurtzite $\mathrm{ZnO}$ single crystals introduced at elevated temperatures. J. Appl. Phys. 2008, 104, 073515. [CrossRef]

9. Carlsson, L.; Svensson, C. Photoplastic effect in ZnO. J. Appl. Phys. 1970, 41, 1652-1656. [CrossRef]

10. Anderson, P.M.; Hirth, J.P.; Lothe, J. Theory of Dislocations, 3rd ed.; Cambridge University Press: New York, NY, USA, 2017. 
11. Osipiyan, Y.A.; Smirnova, I.S. Partial dislocations in the wurtzite lattice. J. Phys. Chem. Solids 1971, 32, 1521-1530. [CrossRef]

12. Hirsch, P.B. The structure and electrical properties of dislocations in semiconductors. J. Microsc. 1980, 118, 3-12. [CrossRef]

13. Holt, D.B.; Yacobi, B.G. Extended Defects in Semiconductors; Cambridge University Press: Cambridge, UK, 2007.

14. Nakamura, A.; Mizoguchi, T.; Matsunaga, K.; Yamamoto, T.; Shibata, N.; Ikuhara, Y. Periodic nanowire array at the crystal interface. ACS Nano 2013, 7, 6297-6302. [CrossRef] [PubMed]

15. Furushima, Y.; Nakamura, A.; Tochigi, E.; Ikuhara, Y.; Toyoura, K.; Matsunaga, K. Dislocation structures and electrical conduction properties of low angle tilt grain boundaries in $\mathrm{LiNbO}_{3}$. J. Appl. Phys. 2016, 120, 142107. [CrossRef]

16. Furushima, Y.; Arakawa, Y.; Nakamura, A.; Tochigi, E.; Matsunaga, K. Nonstoichiometric [012] dislocation in strontium titanate. Acta Mater. 2017, 135, 103-111. [CrossRef]

17. Tochigi, E.; Kezuka, Y.; Nakamura, A.; Nakamura, A.; Shibata, N.; Ikuhara, Y. Direct observation of impurity segregation at dislocation cores in an ionic crystal. Nano Lett. 2017, 17, 2908-2912. [CrossRef] [PubMed]

18. Frank, F.C. Crystal dislocations. Elementary concepts and definitions. Philos. Mag. 1951, 42, 809-819. [CrossRef]

19. Kresse, G.; Furthmüller, J. Efficient iterative schemes for ab initio total-energy calculations using a plane-wave basis set. Phys. Rev. B 1996, 54, 11169. [CrossRef]

20. Blöchl, P.E. Projector augmented-wave method. Phys. Rev. B 1994, 50, 17953. [CrossRef]

21. Perdew, J.P.; Burke, K.; Ernzerhof, M. Generalized gradient approximation made simple. Phys. Rev. Lett. 1996, 77, 3865-3868. [CrossRef] [PubMed]

22. Liechtenstein, A.I.; Anisimov, V.I.; Zaanen, J. Density-functional theory and strong interactions: Orbital ordering in Mott-Hubbard insulators. Phys. Rev. B 1995, 52, R5467-R5470. [CrossRef]

23. Karazhanov, S.Z.; Ravindran, P.; Kjekshus, A.; Fjellvåg, H.; Grossner, U.; Svensson, B.G. Coulomb correlation effects in zinc monochalcogenides. J. Appl. Phys. 2006, 100, 043709. [CrossRef]

24. Nakamura, A.; Ukita, M.; Shimoda, N.; Furushima, Y.; Toyoura, K.; Matsunaga, K. First-principles calculations on slip system activation in the rock salt structure: Electronic origin of ductility in silver chloride. Philos. Mag. 2017, 97, 1281-1310. [CrossRef]

25. Oshima, Y.; Nakamura, A.; Matsunaga, K. Extraordinary plasticity of an inorganic semiconductor. submitted for publication.

26. Bateman, T.B. Elastic Moduli of Single-Crystal Zinc Oxide. J. Appl. Phys. 1962, 33, 3309-3312. [CrossRef]

27. Yan, Y.; Dalpian, G.M.; Al-Jassim, M.M.; Wei, Su-H. Energetics and electronic structure of stacking faults in ZnO. Phys. Rev. B 2004, 70, 193206. [CrossRef]

28. Jhon, M.H.; Glaeser, A.M.; Chrzan, D.C. Computational study of stacking faults in sapphire using total energy methods. Phys. Rev. B 2005, 71, 214101. [CrossRef]

29. Tochigi, E.; Mizoguchi, T.; Okunishi, E.; Nakamura, A.; Shibata, N.; Ikuhara, Y. Dissociation reaction of the $1 / 3<\overline{1} 01>$ edge dislocation in $\alpha-\mathrm{Al}_{2} \mathrm{O}_{3}$. J. Mater. Sci. 2018, in press. [CrossRef]

(C) 2018 by the authors. Licensee MDPI, Basel, Switzerland. This article is an open access article distributed under the terms and conditions of the Creative Commons Attribution (CC BY) license (http:/ / creativecommons.org/licenses/by/4.0/). 